\title{
Escala de Satisfação com a Vida: Evidências de validade e precisão junto de universitários portugueses
}

\section{Satisfaction with Life Scale: Evidences of validity and reliability among Portuguese college students}

\author{
Caroline Reppold (D*, Vanessa Kaiser (D*, Cristian Zanon (D**, Claudio Hutz (D**, Joana R. Casanova (D)**, \\ Leandro S. Almeida (D**** \\ * Universidade Federal de Ciências da Saúde de Porto Alegre, Brasil; **Universidade Federal do Rio Grande do \\ Sul, Brasil; ***Universidade do Minho
}

\begin{abstract}
Resumo
Este artigo apresenta novos dados sobre a precisão e validade dos resultados na Escala de Satisfação com a Vida (SWLS) junto de estudantes universitários. Uma amostra de 545 estudantes provenientes de uma universidade pública portuguesa foi considerada. A unidimensionalidade da escala foi confirmada, apresentando todos os itens cargas fatoriais superiores a .60, ainda que a sua invarância em função do género dos estudantes não se tenha confirmado, desaconselhando comparações de resultados entre homens e mulheres. As correlações moderadas em sentido positivo e negativo com os afetos positivos e negativos avaliados através da Escala de Afetos Positivos e Negativos (PANAS) traduzem evidências de validade de critério. A consistência interna dos resultados nos cindo itens da ESV situou-se em .77, sendo considerada adequada até pelo número reduzido de itens.
\end{abstract}

Palavras-chave: satisfação com a vida, bem-estar psicológico, ensino superior, avaliação psicológica

\begin{abstract}
This paper presents new data on the reliability and validity of results in the Satisfaction With Life Scale (SWLS) among university students. A sample of 545 students from a Portuguese public university was considered. The unidimensionality of the scale was confirmed, with all factor loadings higher than .60, although their invariance according to the gender of the students was not confirmed, and it was not advisable to compare the results
\end{abstract}

\footnotetext{
Caroline Reppold (D) e Vanessa Kaiser (D): Universidade Federal de Ciências da Saúde de Porto Alegre, RS, Brasil.

Cristian Zanon (D) e Claudio Hutz (D): Universidade Federal do Rio Grande do Sul, RS, Brasil.

Joana R. Casanova (D) e Leandro S. Almeida (D): Instituto de Educação, Universidade do Minho. Campus de Gualtar, 4710-075 Braga, Portugal.

Correspondência relativa a este artigo: Leandro Almeida - leandro@ie.uminho.pt
} 
between men and women. Moderate positive and negative correlations with positive and negative affects assessed through the Positive and Negative Affect Schedule (PANAS) translate evidence of criterion validity. The internal consistency of the results in the fifth items of the ESV was .77, considered adequate face the reduced number of items.

Keywords: satisfaction with life, psychological well-being, higher education, psychological evaluation

A satisfação com a vida (SV) corresponde ao componente cognitivo do bem-estar subjetivo (BES), um constructo bastante investigado na atualidade pela Psicologia Positiva (Antaramian, 2017; Barrantes-Brais \& Ureña-Bonilla, 2015; Oliveira, Merino, Privado, \& Almeida, 2017). A SV refere-se ao nível de contentamento que é percebido pelo sujeito quando pensa sobre as várias áreas da sua vida ou a sua vida de forma geral. Trata-se, portanto, de uma avaliação que é subjetiva e envolve uma ponderação sobre o nível de entusiasmo/prazer ou descontentamento/sofrimento que o indivíduo tem ou sente quando pensa no seu modo de viver (Diener, Lucas, \& Oishi, 2009; Muratori, Zubieta, Ubillos, González, \& Bobowik, 2015).

A SV tem impacto na realização dos indivíduos pois traduz maior ou menor envolvimento nas atividades, mais ou menos satisfação com as opções vocacionais e de carreira, maior ou menor percepção de autoeficácia (Cabanach, Arias, Rodríguez, \& Canedo, 2012). Junto dos estudantes do Ensino Superior, traduzirá ainda o sentimento de pertença à instituição e ao curso, o relacionamento com os outros e a percepção de suporte social, estando naturalmente associado com a felicidade, o bem-estar, ou a sintomas de stress, ansiedade e outras psicopatologias (Antaramian, 2017; Barrantes-Brais \& Ureña-Bonilla, 2015; Zanon, 2017; Çivitci, 2015). Estudantes mais felizes apresentam maior satisfação com a vida, inclusive com a sua vida acadêmica, e têm menor probabilidade de abandonar os seus estudos, mostrando maior autonomia e capacidade para persistir nas tarefas acadêmicas, planificar tarefas e cumprir objetivos (Caballero-García \& Sánchez-Ruíz, 2018). Enquanto estudantes, a satisfação com a vida inclui naturalmente as suas vivências acadêmicas, por exemplo a utilização das infraestruturas e recursos, a participação e sucesso no processo de ensino-aprendizagem e os níveis de autonomia e de autoeficácia usufruídos, assim como os projetos de desenvolvimento psicossocial e de carreira em que se encontram implicados (Grebennikov \& Shah, 2013; Magalhães, Machado, \& Sá, 2012; Nauta, 2007).

O principal instrumento usado internacionalmente para avaliar a SV é a Escala de Satisfação com a Vida (Diener, Emmons, Larsen, \& Griffin, 1985). Trata-se de um instrumento composto por cinco itens, respondidos por meio de uma escala ordinal, tipo Likert, de sete pontos, traduzindo o grau de concordância do indivíduo em relação às autoavaliações ou assentimento do sujeito em relação às situações descritas em cada item (por exemplo, ter-se uma vida próxima do ideal, ter condições de vida excelentes, não mudar nada se voltasse a viver de novo). A sua aplicação ocorre frequentemente em conjunto com a avaliação dos afetos positivos e negativos percebidos pelo sujeito, considerados os componentes emocionais do Bem-Estar Subjetivo (Diener, 2000, 2012).

A avaliação da SV requer que o sujeito considere, de forma geral, as suas condições de vida. Nessa avaliação, dependendo das características disposicionais e ambientais, alguns sujeitos tendem a valorizar eventos agradáveis enquanto outros priorizam ou superestimam vivências negativas. Dentre as principais variáveis preditoras dessa avaliação, estão herança genética, traços de personalidade (sobretudo neuroticismo e extroversão), valores, estilos cognitivos e outros atributos, como otimismo e esperança, que podem levar o sujeito a processar e a memorizar as suas experiências e vivências de forma mais otimista, e os faça buscar ativamente aquilo que julgam importante para si (Diener et al., 2009; Marques, Silva, \& Taveira, 2017), procurando a felicidade e evitando as emoções negativas (Caballero-García \& SánchezRuiz, 2018; Matamá et al., 2017).

Sendo estas variáveis associadas relativamente estáveis, a avaliação da SV tende a mostrar resultados pessoais estáveis no tempo. O estudo de Fujita e Diener (2005) confirma a estabilidade do constructo em $76 \%$ de uma 
amostra investigada ao longo de 17 anos na Alemanha. Essa evidência ocorreu mesmo considerando períodos em que os participantes enfrentavam eventos de vida negativos. O estudo de Eid e Diener (2004) evidencia também essa estabilidade, encontrando correlações entre .74 e .84 nos resultados de avaliações de autorrelato realizadas ao longo de 12 semanas.

A Escala de Satisfação com a Vida (ESV) apresenta boas propriedades psicométricas em termos de estrutura fatorial e de consistência interna dos itens. Igualmente os seus resultados apresentam evidências de validade relacionadas a variáveis externas, nomeadamente correlações moderadas ou elevadas com outras medidas de bemestar e com fatores de personalidade (Diener et al., 1985). Estas boas qualidades psicométricas têm sido encontradas em vários países. A título de exemplo, a unidimensionalidade dos itens da escala foi verificada no Brasil (Zanon et al., 2014), Angola (Tomás, Gutiérrez, Sancho, \& Romero, 2015), Chile (Bagherzadeh et al., 2018), Espanha (Vazquez, Duque, \& Hervas, 2013), e Alemanha (Hinz et al., 2018). Uma revisão de 27 artigos publicados com esta escala entre 1985 e 2016, abarcando 66.380 participantes em 24 países, assinala que os participantes, todos oriundos de países ocidentais, atribuem significado semelhante aos itens, independente da cultura ou do sexo, e que a unidimensionalidade da escala foi observada em todos esses estudos (Emerson, Guhn, \& Gadermann, 2017), legitimando estudos transculturais e de género com a escala.

Em Portugal, a ESV foi adaptada inicialmente na década de 1990, em estudos com populações adultas (Neto, Barros, \& Barros, 1990) e com universitários e adultos com profissões diversas (Simões, 1992). Os resultados mantiveram a estrutura fatorial unidimensional e apresentaram evidências de validade convergente esperadas pela literatura e índices adequados de consistência interna. Cite-se que no estudo de Simões, a amplitude da escala Likert foi reduzida de sete para cinco pontos. A escala foi aplicada também junto de professores (Albuquerque \& Lima, 2007) e de gestores empresariais (Martins, Araújo, \& Almeida, 2015), sempre com indicadores psicométricos adequados. Por último, a escala foi também usada num estudo epidemiológico transcultural que investigou a validade da escala para população de idosos (acima de 75 anos de idade) residente em países de língua portuguesa (Humboldt \& Leal, 2017). Os dados da análise fatorial confirmatória indicaram a unidimensionalidade da medida e ampliaram, para a população idosa, as evidências de confiabilidade. No entanto, como se observa, o estudo de adaptação da escala no contexto português ocorreu no início da década de 1990 (Simões, 1992), sendo de interesse a verificação atualizada de evidências de validade da escala junto da população universitária.

O objetivo deste artigo é apresentar dados atualizados sobre o funcionamento da Escala de Satisfação com a Vida junto da população universitária portuguesa. Mais concretamente apresentamos as propriedades psicométricas dos resultados da escala em termos de precisão e de validade (estrutura fatorial e critério externo). Ao mesmo tempo, sendo frequentes análises comparativas de subgrupos de estudantes nas variáveis relacionadas às expectativas e vivências académicas, incluindo diferenciação segundo o género (Diniz et al., 2018; Gil et al., 2013), procura-se verificar neste estudo se é invariante a estrutura dimensional da escala em função do género.

\section{Método}

\section{Participantes}

Os dados foram recolhidos junto de uma amostra de conveniência constituída por estudantes universitários portugueses provenientes de uma universidade pública no norte de Portugal. Todos os estudantes estavam no $1 .^{\circ}$ ano do seu curso, frequentando o primeiro semestre do ano escolar. A amostra do presente estudo foi composta por 545 participantes, sendo $29.4 \%$ homens. A idade dos participantes variou entre 17 e 59 anos, situando-se a média de idades nos 19.5 anos $(D P=4.32)$. Estes estudantes repartem-se por cursos nas áreas de ciências e tecnologia (42.5\%), ciências económicas $(28.0 \%)$ e ciências sociais e humanas (29.5\%). A maioria dos estudantes $(61.0 \%)$ refere estar a frequentar um curso de primeira opção, ao mesmo tempo que $81.3 \%$ referem que estão a frequentar a universidade de sua primeira escolha. Estes dados sugerem que bastantes alunos estão a frequentar a 
universidade de sua primeira opção, mas não o curso (dados do Ministério da Educação apontam que, dado o sistema de numerus clausus no acesso dos estudantes às instituições e cursos do ensino superior, cerca de $60 \%$ dos alunos não estão colocados no par curso e instituição de primeira opção. A larga maioria destes estudantes são provenientes de famílias sem tradição de frequência do Ensino Superior, ou seja apenas $13.5 \%$ referem que o seu pai frequentou a universidade (17.6\% das mães). Estas caraterísticas sociofamiliares dos estudantes se entendem tratando-se de uma universidade pública instalada no norte de Portugal numa região marcadamente agrícola e rural.

\section{Instrumentos}

Os instrumentos utilizados neste estudo foram a Escala de Satisfação com a Vida desenvolvida por Diener, Emmons, Larsen e Griffin (1985), e adaptada para língua portuguesa por Zanon et al., 2014. É composta por cinco itens de autorrelato, cujo conteúdo avalia o nível de satisfação dos sujeitos. As respostas são dadas por meio de uma escala Likert de cinco pontos que variam entre "discordo plenamente" e "concordo plenamente". Para avaliação da validade convergente, foi utilizada também a Escala de Afetos Positivos e Negativos (PANAS) adaptada para português por Reppold et al. (prelo), composta por 20 itens que se repartem por dois fatores: afetos positivos e afetos negativos. Os itens são constituídos por adjetivos (ex. aflito, animado) com chaves de respostas em uma escala Likert de cinco pontos que variam de "nem um pouco" a "extremamente". A escala foi desenvolvida e validada com base na escala PANAS (Positive Affects and Negative Affects Scale) de Watson, Clark e Tellegen (1988), apresentando índices adequados de consistência interna (.88 e .84) e evidências de validade.

\section{Procedimento}

A realização do presente estudo considerou os princípios éticos para a investigação com seres humanos. A recolha de dados ocorreu em contexto de sala de aula, durante o tempo letivo cedido pelos professores, preferencialmente no início ou no final das aulas. Os estudantes foram convidados a participar no estudo, explicando-se os objetivos e assegurando-se a confidencialidade das suas respostas e o direito à não-participação.

A fim de verificar a validade de estrutura interna do instrumento, foi realizada uma Análise de Componentes Principais com o programa IBM/SPSS versão 23.0. A precisão da escala foi analisada através do coeficiente Alfa de Cronbach. A análise fatorial confirmatória dos itens e a análise da sua invariância segundo o género dos alunos foram realizadas através do programa MPlus, versão 6 (Muthén \& Muthén, 2010).

A análise invariância da medida permite testar diferentes níveis de invariância do modelo de interesse entre diferentes grupos, no caso concreto deste estudo tomando o género dos estudantes. O nível menos restritivo (e.g., configural) avalia a estrutura do modelo considerando o número de fatores e se os itens saturam nestes fatores. Se este modelo recebe suporte, testa-se o modelo métrico que assume a mesma estrutura nos modelos (e.g., nível configural), mas também que as cargas fatoriais são similares. Se o modelo métrico for adequado, testa-se o nível escalar, que assume mesma estrutura (e.g., nível configural), mesmo padrão de cargas fatoriais (e.g., nível métrico) e que os interceptos (e.g., média dos itens) são similares. A realização de comparações de médias é adequada apenas se o nível escalar for adequado. Se houver evidência de invariância métrica, é possível se comparar modelos através de correlações/regressões ou structural equation modeling. Antecipa-se que, quando apenas o nível configural apresenta evidências, as comparações de grupos podem ser inapropriadas e levar a conclusões enviesadas (Byrne \& Van de Vijver, 2010).

\section{Resultados}

Procedeu-se a uma Análise de Componentes Principais com os cinco itens da ESV, observando-se previamente os índices de fatorização dos itens: índice KMO de .79 e teste de esfericidade de Barlett significativo $\left(\chi^{2}=649.80\right.$, 
$g l=10, p<0.001$ ), indicando a possibilidade de fatoração da matriz de dados. Os resultados obtidos indicaram uma solução unifatorial, explicando o componente retido $53.7 \%$ da variância dos itens. A carga fatorial, a média e o desvio-padrão obtidos para cada item estão apresentados na Tabela 1. Em relação à fidedignidade, a análise do alfa de Cronbach indicou que a escala apresenta índice adequado de consistência interna dos itens $(\alpha=.77)$.

Tabela 1

Carga fatorial, média e desvio-padrão de cada item da ESV

\begin{tabular}{lcccc}
\hline & & \multicolumn{3}{c}{ Carga fatorial } \\
Itens & $M$ & $D P$ & PE & EO \\
\hline Vida próxima do ideal & 2.79 & .76 & .73 & .84 \\
Condições de vida excelentes & 3.11 & .78 & .71 & .77 \\
Satisfação com a vida & 3.11 & .80 & .84 & .83 \\
Atingir as coisas importantes & 3.22 & .81 & .74 & .72 \\
na vida & & & & \\
Nada mudaria na vida & 2.72 & .83 & .62 & .61 \\
\hline
\end{tabular}

Nota $: \mathrm{PE}=$ Presente estudo $; \mathrm{EO}=$ Escala original

Como podemos observar, a média das pontuações nos cinco itens aproximam-se de um valor intermédio tomando a amplitude de 1 a 5 pontos na escala usada, aproximando-se o desvio-padrão da unidade. Ao mesmo tempo, as saturações dos itens no único fator isolado são superiores a .60, reforçando a unidimensionalidade dos itens. Neste sentido, procedeu-se a uma análise fatorial confirmatória (AFC) usando o método Maximum Likelihood com erros-padrões robustos (MLR), registando-se bons índices de ajustamento para a solução unifatorial da escala (Figura 1): $\chi 2(5)=11, p>.05 ; \mathrm{CFI}=.98 \mathrm{e}$ RMSEA $=.06($ IC 95\% $=.00-.09)$. Na Tabela 2, apresentam-se os resultados do teste da invariância deste modelo tomando o género dos alunos.

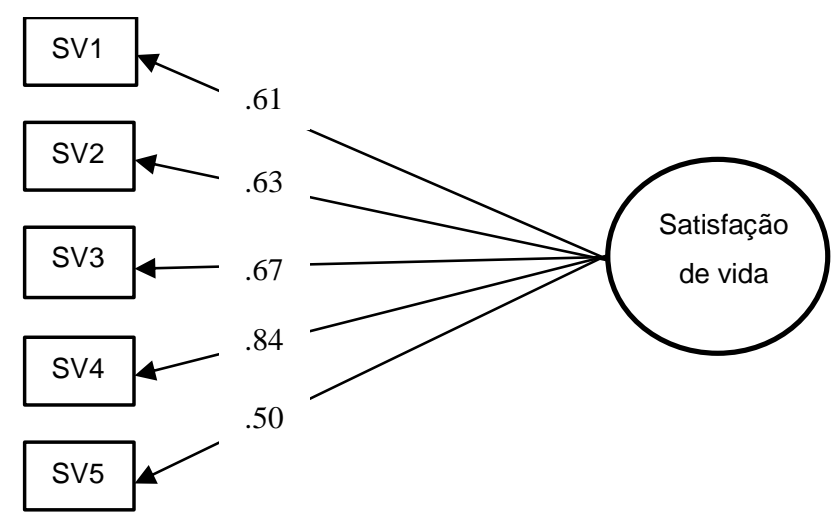

Figura 1. Representação gráfica do modelo unidimensional testado na AFC (MLR)
Tabela 2

Análise fatorial confirmatória multigrupo (sexo) da Escala de Satisfação com a Vida

\begin{tabular}{lcccccc}
\hline $\begin{array}{l}\text { Níveis de } \\
\text { invariância }\end{array}$ & $\chi^{2}$ & $g l$ & CFI & RMSEA & $\begin{array}{c}\text { RMSEA } \\
\text { (IC 95\%) }\end{array}$ & NParam \\
\hline Configural & 20 & 10 & .979 & .063 & $.02-.10$ & 30 \\
Métrico & 33 & 14 & .957 & .075 & $.04-.11$ & 26 \\
Escalar & 38 & 18 & .957 & .067 & $.04-.10$ & 22 \\
\hline
\end{tabular}

Nota. $\chi 2=$ chi-quadrado, $g l=$ graus de liberdade, $\mathrm{CFI}=$ Comparative Fit Index, RMSEA = Root Mean Square Error of Approximation, NParam = Número de parâmetros livremente estimados

A análise fatorial confirmatória multigrupo entre homens e mulheres indicou nível de invariância apenas configural, sugerindo os resultados nesta amostra que não são comparáveis os resultados de homens e mulheres. A variação superior a .01 no CFI suporta o modelo menos restritivo (configural), sugerindo confirmações em novas amostras. Finalmente, para efeitos de evidências de validade de critério, na Tabela 3 apresentam-se as correlações encontradas entre a satisfação com a vida (ESV) e os afetos positivos e afetos negativos (PANAS).

Tabela 3

Correlações entre Satisfação com a Vida e Afetos Positivos e Negativos

\begin{tabular}{lcccc}
\hline Variáveis & $M$ & $D P$ & SV & AP \\
\hline Satisfação com a vida & 14.49 & 2.97 & & \\
Afetos positivos & 32.60 & 6.92 & $.36^{* * *}$ & \\
Afetos Negativos & 19.79 & 5.23 & $-.25^{* * *}$ & $-.18^{* *}$ \\
\hline$* *$ & & & &
\end{tabular}

$* * p<.01 ; * * * p .001$

Como podemos verificar, os resultados na ESV apresentam-se moderadamente correlacionados com os Afetos Positivos, e como seria esperado em sentido positivo. Menor correlação se encontra entre ESV e Afetos Negativos, sendo relevante destacar o sentido negativo dessa correlação e o facto da sua magnitude ser mais elevada que cruzando as duas dimensões de afetos. 


\section{Discussão}

O propósito desse artigo foi apresentar dados de precisão e evidências de validade dos resultados na Escala de Satisfação com a Vida (ESV; Zanon et al., 2014) em uma amostra de universitários portugueses. Dentre os principais resultados, verificou-se que o instrumento apresentou propriedades psicométricas adequadas que suportam a sua utilização no contexto universitário português. Mais especificamente, observou-se que a escala com cinco itens manteve sua estrutura unidimensional, como aconteceu em pesquisas noutros países (Bagherzadeh et al., 2018; Tomás et al., 2015; Zanon et al., 2014). A variância dos cinco itens explicada por este único fator situou-se em $53.7 \%$, valor um pouco inferior à variância explicada pelo único fator no estudo original com a escala (66\%), mas semelhante ao encontrado em outros países (Diener, 2012; Emerson et al., 2017). As cargas fatoriais dos itens também apresentam valores satisfatórios e próximos aos valores encontrados no estudo original americano. O único item que apresentou carga levemente inferior no contexto português foi o item 1 que trata da percepção geral sobre se a vida está próxima do ideal. Este resultado evidencia que este item tem alguma especificidade no contexto português face ao contexto americano (Diener et al., 1985), podendo esta situação ter duas interpretações. Por um lado, sendo o primeiro item da escala, esse valor pode refletir alguma hesitação dos sujeitos na sua autoavaliação quando iniciam o preenchimento da escala. Ao mesmo tempo, e reportando-nos à população portuguesa, pode ser difícil fixar, por razões culturais, a vida ideal ou delimitá-la por algum saudosismo, pessimismo ou moderação instituídos socioculturalmente. Este último ponto pode, aliás, explicar a não invariância do modelo fatorial da escala tomando homens e mulheres da amostra para além do nível básico (configural).

Em relação a invariância do modelo entre homens e mulheres, a invariância configural sugere que a estrutura do modelo é semelhante entre homens e mulheres, mas o padrão de cargas fatoriais não é equivalente nos dois grupos. Considerando que a carga fatorial representa um coeficiente de regressão, diferenças de cargas fatoriais indicam que o fator latente (e.g., satisfação de vida) explica diferentes proporções de variância nos mesmos itens entre os grupos, o que pode estar associado a algum viés metodológico para algum grupo (e.g., aquiescência ou desejabilidade social) (Byrne \& Van de Vijver, 2010). Este resultado indica que a comparação de satisfação de vida entre homens e mulheres pode produzir resultados enviesados, não sendo recomendada. Diferente deste resultado, Zanon et al. (2014) encontrou evidências de invariância escalar de satisfação de vida no Brasil entre homens e mulheres com a mesma escala, sendo importante aprofundar esta questão em Portugal com amostras universitárias mais heterogéneas e representativas, até porque frecuentemente se comparam homens e mulheres nos seus níveis de satisfação com a vida.

Ainda em termos de validade dos resultados da ESV, as correlações obtidas com a medida dos afetos positivos e afetos negativos (PANAS) foram de magnitude moderada, mas sempre no sentido positivo ou negativo consoante a tonalidade dos afetos avaliados. Os coeficientes de correlação obtidos, em termos de magnitude e de sentido, aproximam-se dos valores obtidos em estudos anteriores (Humboldt \& Leal, 2017; Zanon et al., 2014) e, neste sentido, suportam evidências de validade de critério. Em ambos os instrumentos, as autoavaliações dos estudantes sugerem níveis maiores de satisfação com a vida por parte dos que experienciam ou pontuam mais elevado nos afetos positivos, e vice-versa em relação aos afetos negativos.

A fiabilidade dos resultados da escala na presente amostra situou-se em níveis adequados (alfa de Cronbach igual a .77), sobretudo considerando-se o reduzido número de itens da escala e também porque a pontuação dos sujeitos é feita agora numa escala Likert de 5 e não de 7 pontos como na versão original americana que permitia maior variabilidade de valores. O índice de consistência interna obtido foi próximo ao encontrado noutros estudos com amostras portuguesas (Marques, Silva, \& Taveira, 2017; Neto, Barros, \& Barros, 1990; Simões, 1982).

Finalmente, mesmo que os dados apresentados sugiram que a escala possui propriedades psicométricas (validade, precisão) que viabilizam seu o uso e que são próximas aos resultados obtidos em estudos internacionais, importa atender a algumas limitações metodológicas deste estudo. Desde logo, a amostra não é suficientemente 
abrangente e representativa dos estudantes universitários portugueses pois que recolhida, apenas, junto de uma universidade. Por outro lado, a opção tomada de utilizar uma escala Likert de 5 pontos, e não 7 como noutras versões usadas em diversas pesquisas, dificulta uma comparação linear dos resultados obtidos entre os estudos referenciados. A auscultação dos estudantes sobre uma autoavaliação mais precisa recorrendo a 5 ou 7 níveis da escala pode ajudar a decidir neste ponto, podendo inclusive esta opção metodológica influir na invariância do modelo estrutural da escala quando se consideram diferentes grupos de estudantes. Ao mesmo tempo, importa aprofundar a origem da especificidade do item 1 e se essa especificidade pode ajudar a compreender e a ultrapassar a não verificação da invariância do modelo fatorial segundo o género, até porque na investigação com estudantes do ensino superior frequentemente se procura verificar se homens e mulheres diferem nas variáveis descritivas da sua adaptação e sucesso académico.

\section{Referências}

Antaramian, S. (2017). The importance of very high life satisfaction for students' academic success. Cogent Education, 4(1). https://doi.org/10.1080/2331186X.2017.1307622

Albuquerque, I., \& Lima, M. (2007). Personalidade e bem-estar subjetivo: Uma abordagem com os projectos pessoais. O Portal dos Psicólogos. Recuperado de http://www.psicologia.pt/artigos/textos/A0373.pdf

Bagherzadeh, M., Loewe, N., Mouawad, R. G., Batista-Foguet, J. M., Araya-Castillo, L., \& Thieme, C. (2018). Spanish version of the Satisfaction with Life Scale: Validation and factorial invariance analysis in Chile. Spanish Journal of Psychology, 21, e2. https://doi.org/10.1017/sjp.2018.2

Barrantes-Brais, K., \& Ureña-Bonilla, P. (2015). Bienestar psicológico y bienestar subjetivo en estudiantes universitarios costarricenses. Revista Intercontinental de Psicología y Educación, 17(1), 101-123. Recuperado de http://www.redalyc.org/articulo.oa?id=80242935006

Byrne, B. M., \& Van de Vijver, F.R. (2010). Testing for measurement and structural equivalence in large-scale cross-cultural studies. International Journal of Testing, 10(2), 107-132. https://doi.org/10.1080/ 15305051003637306

Caballero-García, P. A., \& Sánchez Ruiz, S. (2018). La felicidad en estudiantes universitarios. ¿Existen diferencias según género, edad o elección de estudios? Revista Electrónica Interuniversitaria de Formación del Profesorado, 21(3). https://doi.org/10.6018/reifop.21.3.336721

González-Cabanach, R., Valle-Arias, A., Freire-Rodríguez, C., \& Ferradás-Canedo, M. (2012). The relations between perceived self-efficacy and psychological well-being in university students. Revista Mexicana de Psicología, 29(1), 40-48. Recuperado de http://www.redalyc.org/articulo.oa?id=243030189004

Çivitci, A. (2015). Perceived stress and life satisfaction in college students: Belonging and extracurricular participation as moderators. Procedia-Social and Behavioral Sciences, 205, 271-281. https://doi.org/ 10.1016/j.sbspro.2015.09.077

Diener, E. (2000). Subjective well-being: The science of happiness and a proposal for a national index. American Psychologist, 55(1), 34-43. https://doi.org/10.1037/0003-066X.55.1.34

Diener, E. (2012). New findings and future directions for subjective well-being research. American Psychologist, 67(8), 590-597. https://doi.org/10.1037/a0029541

Diener, E., Emmons, R. A., Larsen, R. J., \& Griffin, S. (1985). The satisfaction with life scale. Journal of Personality Assessment, 49(1), 71-75. https://doi.org/10.1207/s15327752jpa4901_13

Diener, E., Lucas, R. E., \& Oishi, S. (2009). Subjective well-being: The science of happiness and life satisfaction. In C. R. Snyder, \& S. J. Lopez (Eds.), Oxford Handbook of Positive Psychology (2a ed.) (pp. 187-194). Oxford: Oxford University Press. https://doi.org/10.1093/oxfordhb/9780195187243.013.0017

Diniz, A. M., Alfonso, S., Araújo, A. M., Deaño, M., Costa, A. R., Conde, A., \& Almeida, L. S. (2018). Gender 
differences in firstyear college students' academic expectations, Studies in Higher Education, 43(4), 689-701. https://doi.org/10.1080/03075079.2016.1196350

Eid, M., \& Diener, E. (2004). Global judgments of subjective well-being: Situational variability and long-term stability. Social Indicators Research, 65, 245-277. https://doi.org/10.1023/B:SOCI.0000003801.89195.bc

Emerson, S. D., Guhn, M., \& Gadermann, A. M. (2017). Measurement invariance of the Satisfaction with Life Scale: Reviewing three decades of research. Quality of Life Research, 26, 2251-2264. https://doi.org/ 10.1007/s11136-017-1552-2

Fujita, F., \& Diener, E. (2005). Life satisfaction set-point: Stability and change. Journal of Personality and Social Psychology, 88, 158-164. https://doi.org/10.1037/0022-3514.88.1.158

Gil, S. A., Deaño, M. D., Rodríguez, A. C., Costa, A. R., Araújo, A. M., \& Almeida, L. S. (2013). Perfiles de expectativas académicas en alumnos españoles y portugueses de enseñanza superior. Revista GalegoPortuguesa de Psicoloxía e Educación, 21(1), 125-136. http://hdl.handle.net/2183/12610

González-Cabanach, R., Valle-Arias, A., Freire-Rodríguez, C., \& Ferradás-Canedo, M. (2012). The relations between perceived self-efficacy and psychological well-being in university students. Revista Mexicana de Psicología, 29(1), 40-48. Recuperado de http://www.redalyc.org/articulo.oa?id=243030189004

Gouveia, V., Milfont, T., Fonseca, P. N., \& Coelho, J. P. M. (2009). Life satisfaction in Brazil: Testing the psychometric properties of the Satisfaction with Life Scale (SWLS) in five Brazilian samples. Social Indicators Research, 90, 267-277. https://doi.org/10.1007/s11205-008-9257-0

Grebennikov, L., \& Shah, M. (2013). Monitoring trends in student satisfaction. Tertiary Education and Management, 19(4), 301-322. https://doi.org/10.1080/13583883.2013.804114

Hinz, A., Conrad, I., Schroeter, M. L., Glaesmer, H., Brähler, E., Zenger, M., Kocalevent, R. D., \& Herzberg, P. Y. (2018). Psychometric properties of the Satisfaction with Life Scale (SWLS), derived from a large German community sample. Quality of Life Research, 27(6), 1661-1670. https://doi.org/10.1007/s11136-018-1844-1

Humboldt, S., \& Leal, I. (2017). A health-related satisfaction with life scale measure for use with cross-national older adults: A validation study. Review of European Studies, 9(3). https://doi.org/10.5539/res.v9n3p21

Magalhães, A., Machado, M. L., \& Sá, M. J. (2012). Satisfação dos estudantes do ensino superior português. Matosinhos: CIPES.

Martins, M., Araújo, A., \& Almeida, L. (2015). Personalidade e bem-estar subjetivo na excelência profissional: Validação de escalas junto de gestores portugueses. Revista E-Psi, 5(2), 86-102. https://revistaepsi.com/ artigo/2015-ano5-volume2-artigo5/

Matamá, J., Mendes, R., Pinho-Pereira, S., Nascimento, D., Campina, A., \& Costa-Lobo, C. (2017). Bem-Estar Subjetivo: Uma revisão narrativa da literatura. Revista de Estudios e Investigación en Psicología y Educación, 7, 243-246. https://doi.org/10.17979/reipe.2017.0.05.2664

Muratori, M., Zubieta, E., Ubillos, S., González, J. L., \& Bobowik, M. (2015). Felicidad y Bienestar Psicológico: Estudio comparativo entre Argentina y España. Psykhe (Santiago), 24(2), 1-18. https://doi.org/10.7764/ psykhe.24.2.900

Muthén, L. K., \& Muthén, B. O. (2010). Mplus User's Guide (6th ed.). Los Angeles, CA: Muthén \& Muthén..

Nauta, M. M. (2007). Assessing college students' satisfaction with their academic majors. Journal of Career Assessment, 15, 446-462. https://doi.org/10.1177/1069072707305762

Neto, F. (1993). The satisfaction with life scale: Psychometrics properties in an adolescent sample. Journal of Youth and Adolescence, 22(2), 125-134. https://doi.org/10.1007/BF01536648

Neto, F., Barros, J., \& Barros, A. (1990). Satisfação com a vida. In L. Almeida, R. Santiago, P. Silva, O. Caetano, \& J. Marques (Eds.), A Acção educativa: Análise psicossocial (pp. 105-117). Leiria: ESEL/APPORT.

Oliveira, E. P., Merino, M. D., Privado, J., \& Almeida, L. S. (2017). Funcionamento psicológico positivo numa 
amostra portuguesa de estudantes. Revista de Estudios e Investigación en Psicología y Educación, (07), 93-96. https://doi.org/10.17979/reipe.2017.0.07.2733

Reppold, C., Kaiser, V., Zanon, C., Hutz, C., \& Almeida, L. (prelo). Escala de Satisfação de Vida: Evidências de validade, precisão e atualização de normas para em uma amostra portuguesa.

Simões, A. (1992). Ulterior validação de uma escala de satisfação com a vida (SWLS). Revista Portuguesa de Pedagogia, 3, 503-515.

Tomás, J. M., Gutiérrez, M., Sancho, P., \& Romero, I. (2015). Measurement invariance of the Satisfaction with Life Scale (SWLS) by gender and age in Angola. Personality and Individual Differences, 85, 182-186. https://doi.org/10.1016/j.paid.2015.05.008

Vazquez, C., Duque, A., \& Hervas, G. (2013). Satisfaction with life scale in a representative sample of Spanish adults: Validation and normative data. Spanish Journal of Psychology, 16, e82. https://doi.org/10.1017/ sjp. 2013.82

Zanon, C. (2017). Felicidade e indicadores de psicopatologia em brasileiros. Avaliaçao Psicologica: Interamerican Journal of Psychological Assessment, 16(4), 452-457. https://doi.org/10.15689/ap.2017. 1604.12857

Zanon, C., Bardagi, M. P., Layous, K., \& Hutz, C. S. (2014). Validation of the Satisfaction with Life Scale to Brazilians: Evidences of measurement noninvariance across Brazil and US. Social Indicators Research, 119(1), 443-453. https://doi.org/10.1007/s11205-013-0478-5

Watson, D., Clark, L. A., \& Tellegen, A. (1988). Development and validation of brief measures of positive and negative affect: the PANAS scales. Journal of Personality and Social Psychology, 54, 1063-1070. https://doi.org/10.1037//0022-3514.54.6.1063

Fecha de recepción: 25 de octubre de 2018.

Fecha de revisión: 20 de abril de 2019.

Fecha de aceptación: 17 de mayo de 2019.

Fecha de publicación: 1 de julio de 2019. 